\title{
Incidence of Neuropathy with Weekly Paclitaxel and Role of Oral Glutamine Supplementation for Prevention of Paclitaxel Induced Peripheral Neuropathy Randomized Controlled Trial
}

\begin{abstract}
Background: Peripheral neuropathy is damage to the peripheral nerve. The most common cause of neuropathy is paclitaxel. Several avenues have been explored to ameliorate the neurotoxicity associated with paclitaxel. Clinical studies have assessed the efficacy of glutamine with different doses and schedules to prevent gastrointestinal toxicity (mucositis, diarrhea) and peripheral neuropathy in patients receiving a variety of chemotherapy agents or radiation therapy and found that glutamine can prevent paclitaxel-induced peripheral neuropathy. Methods: Total of 50 patients, aged 30 or more with diagnosis of cancer and fulfilling the inclusion and exclusion criteria, formed the study population. We assigned 25 patients to the glutamine group and 25 patients to no glutamine group. All patients received weekly paclitaxel. Results: The incidence of neuropathy of all grades at 3 months was $78 \%$ and at 6 months was $80 \%$.In this study, most common symptom reported was numbness in toes $(74 \%)$. In this study, Grade 1 was the most common grade of symptom reported by the patient $(40 \%-50 \%)$. 2nd, 3rd, and 4 th most common grade of symptom reported by the patient was Grade 0 , Grade 2, and Grade 3, respectively. There was no Grade 4 symptom reported by any patient. All the symptoms were statistically comparable in both groups (Myalgias: $P=0.066$, Arthralgia: $P=0.93$, Dysesthesia: $P=0.82$, Paresthesia: $P=0.92$, Numbness fingers: $P=0.97$, Numbness toes: $P=0.60)$. In our study, there was no incidence of cranial nerve weakness or any incidence of the postural drop. The electrophysiological study is the best tool available and can detect neuropathy at the very earlier stage even when the clinical exam is negative. Apart from that nature of neuropathy can be determined but grading is not possible which makes very difficult to decide on follow-up examinations when the physician should intervene. Moreover, there are fluctuations in SNAP and CMAP, and these fluctuations are most probably related to the innate variability of serial nerve conduction study parameters, particularly motor and sensory amplitude. Glutamine did not prevent neurotoxicity induced by weekly paclitaxel.
\end{abstract}

Keywords: Glutamine supplementation for prevention of paclitaxel induced neuropathy, Incidence of neuropathy with weekly paclitaxel, Paclitaxel induced neuropathy

\section{Introduction}

Peripheral neuropathy is damage to the peripheral nerve. Chemotherapy-induced peripheral neuropathy (CIPN) can be due to treatment with multiple chemotherapeutic agents, including platinum compounds (cisplatin, carboplatin, and oxaliplatin), vinca alkaloids (vincristine, vinblastine), taxanes (paclitaxel, docetaxel), thalidomide, and bortezomib. Peripheral neuropathy is a common nonhematological side effect of these drugs. The mechanisms of this neuropathy are usually attributed to microtubule disruption (taxanes, Vinca alkaloids) or a direct toxic effect platinum

This is an open access journal, and articles are distributed under the terms of the Creative Commons Attribution-Non Commercial-ShareAlike 4.0 License, which allows others to remix, tweak, and build upon the work non-commercially, as long as appropriate credit is given and the new creations are licensed under the identical terms.

For reprints contact: reprints@medknow.com compounds. ${ }^{[1]}$ The most common cause of neuropathy is paclitaxel. It is dose and infusion duration related. Neuropathy risk is more in patients who have other comorbidities (such as diabetes or kidney disease) or who have been previously treated with other neurotoxic chemotherapy such as cisplatin and vincristine $\mathrm{e}^{[2,3]}$

Neurotoxicity to both small-sensory and large-sensory fibers is seen. ${ }^{[4]}$ It is a sensory predominant neuropathy. Motor neuropathy has been observed with higher doses of paclitaxel. ${ }^{[5]}$ Paclitaxel causes more neuropathy when infused over $3 \mathrm{~h}$ as compared to $24 \mathrm{~h}$ infusion. ${ }^{[6,7]}$ Moreover, dose-dense schedule of weekly paclitaxel

How to cite this article: Abbas W, Rao RR,
Agarwal A, Saha R, Bajpai P, Qureshi S, et al.
Incidence of neuropathy with weekly paclitaxel
and role of oral glutamine supplementation for
prevention of paclitaxel induced peripheral neuropathy
randomized controlled trial. Indian J Med Paediatr
Oncol 2018;39:339-48.

How to cite this article: Abbas $W$, Rao RR, Agarwal A, Saha R, Bajpai P, Qureshi S, et al. Incidence of neuropathy with weekly paclitaxel and role of oral glutamine supplementation for prevention of paclitaxel induced peripheral neuropathy Oncol 2018;39:339-48.

\section{Waseem Abbas, Ranga Raju Rao, Amit Agarwal, Rajat Saha, Peush Bajpai, Suhail Qureshi, Aditi Mittal}

NBE (National Board of Examinations), Ministry of Health and Family Welfare, Dwarka, New Delhi, India

Address for correspondence Dr. Waseem Abbas,

Lajpat Nagar-1, B-Block,

143/144, First Floor - 110024 ,

New Delhi, India.

E-mail:drabbasdoc@gmail.com

Access this article online

Website: www.ijmpo.org

DOI: 10.4103/ijmpo.ijmpo_38_17

Quick Response Code:

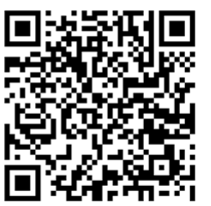


causes more neuropathy than 3 weekly. Incidence is about $75 \%$. The neuropathy with weekly paclitaxel is generally a sensory polyneuropathy affecting large fibers, can also lead to cranial nerve palsies, motor weakness, and autonomic dysfunction. ${ }^{[8]}$ The options of stopping treatment early or dose reducing are equally undesirable in the advanced disease setting, but may have greater implications in the adjuvant setting because taxanes have become part of the standard treatment for a wide variety of neoplasm, including breast, ovary, lung, and gastrointestinal tumors, first line as well as subsequent therapy.

Several avenues have been explored to ameliorate the neurotoxicity associated with paclitaxel, including the use of nonsteroidal anti-inflammatory agents, corticosteroids, and amifostine and these treatments have been uniformly unsuccessful. ${ }^{[9]}$ Savarese et $a .^{[10]}$ reported the successful reduction of paclitaxel-associated myalgias and arthralgias by glutamine in five patients treated with paclitaxel doses ranging from 175 to $200 \mathrm{mg} / \mathrm{m}^{2}$. All of the patients had debilitating paclitaxel-associated myalgias/arthralgias associated with their first cycle of therapy. For subsequent cycles, they received glutamine (10 g p. o. t. i. d.) 3 for 4 days starting $24 \mathrm{~h}$ after the completion of paclitaxel. No patient had a recrudescence of symptoms while on glutamine.

Glutamine is a neutral gluconeogenic nonessential amino acid stored primarily in skeletal muscle $(75 \%)$ and liver (25\%). Among its many functions, glutamine serves as the primary carrier of nitrogen between tissues. It is also the main energy source for rapidly proliferating cells such as intestinal epithelium, activated lymphocytes, and fibroblasts. Glutamine is depleted in stress states such as major surgery, sepsis, and cancer. ${ }^{[11]}$ It is also essential for maintenance of gut epithelium for patients on total parenteral nutrition as its omission hastens villous atrophy. ${ }^{[12]}$ Preclinical data suggest that glutamine supplementation does not augment tumor cell growth and may augment response to chemotherapy.

Clinical studies have assessed the efficacy of glutamine with different doses and schedules to prevent gastrointestinal toxicity (mucositis, diarrhea) and peripheral neuropathy in patients receiving a variety of chemotherapy agents or radiation therapy and found that glutamine can prevent paclitaxel-induced peripheral neuropathy. Most robust data on prevention of paclitaxel-induced neuropathy is the study done by Stubblefield et al. ${ }^{[13]}$ However, there are no studies on prevention of paclitaxel in India and no randomized trial has been done to look for the incidence of neuropathy with weekly paclitaxel as compared to 3 weekly.

The objective of this randomized study was to evaluate the incidence, clinical presentation of paclitaxel-induced neuropathy and whether it can be prevented by glutamine. Further effort was made to see whether electrophysiological studies can be used to quantify paclitaxel-induced neuropathy.

\section{Methods and Materials}

From April 2013 to November 2014, a total of 50 patients with histologically confirmed malignancies treated at B. L Kapur Superciality hospital, NewDelhi, India were enrolled in the study.

\section{Study design}

This was randomized controlled trial.

Randomization was done with a total of 50 opaque, sealed envelopes, containing an identifier for glutamine group (Group 1) and containing an identifier for control group (Group 2), shuffled. The order of the shuffled envelopes determined the allocation of participants to treatments.

Patients were randomly assigned to one of the treatment arms.

- Glutamine group: (Group 1)

- Control group: (Group 2).

All patients enrolled received weekly paclitaxel as per indications. The dose of paclitaxel was $80 \mathrm{mg} / \mathrm{m}^{2}$ intravenous over $1 \mathrm{~h}$ weekly.

All patients enrolled in glutamine group (Group 1) received oral glutamine $15 \mathrm{~g}$ daily (that is approximately $0.25 \mathrm{mg} / \mathrm{kg}$ ) for 6 months. Glutamine was provided free of cost to all the patients for the entire duration of treatment.

Moreover, patients from the CONTROL group (Group 2) did not receive glutamine or any kind of supplementation apart from routine medications [Figure 1].

\section{Sample size}

A total of 50 patients were included ( 25 patients for each group).

Sample size calculation was based on the study by Linda Vehdat et al. ${ }^{[27]}$ "Reduction of Paclitaxel-induced Peripheral Neuropathy with Glutamine" that showed numbness in toes was reduced by $40 \%$ in glutamine arm (intervention arm). Thus, assuming minimum $40 \%$ change is clinically relevant to show the effectiveness of the intervention, a sample size of total of 50 patients and 25 patients in each group was calculated. All calculation was done by assuming an error of $\alpha=5 \%$ (probability of type I error) to achieve $\beta=80 \%$ power of the study. Calculation based on this formula: $\mathrm{n}=\mathrm{f}(\alpha / 2$, $\beta) \times(\mathrm{p} 1 \times[100-\mathrm{p} 1]+\mathrm{p} 2 \times[100-\mathrm{p} 2]) /(\mathrm{p} 2-\mathrm{p} 1) 2$.

\section{Inclusion criteria}

- Patients eligible for paclitaxel therapy as per indications

- Performance status (ECOG)-0-2

- Age $>18$ and $<80$ years

- Patients with established malignancies by histopathological examination report

- Written informed consent.

\section{Exclusion criteria}

- Central nervous system metastases 
- Prior treatment with taxanes

- Severe hypersensitivity to taxanes

- Compromised organ function

- Baseline neuropathy

- Life expectancy $<6$ months (clinical judgment)

- Abnormal hematopoietic function. Total leukocyte count $<3000$, platelet count $<100,000$

- Pregnant and lactating mothers.

Written informed bilingual consent was taken from all the subjects. This study has been approved by the ethical committee (clinical) and scientific committee of Dr. B. L. Kapur Superciality Hospital.

Baseline complete blood investigations were done including complete hemogram, liver function tests, kidney function tests to see fitness for chemotherapy. Vitamin B-12 and folate levels were also done. If Vitamin B-12 and folate levels below the reference range below the reference range were replaced.

\section{Data collection methods}

Data were collected by direct observation, clinical examination, and electrodiagnostic studies.

Data were recorded for each patient using a structured pro forma which included.

\section{Neurologic evaluation}

- The detailed neurological examination was done before the start of paclitaxel and thereafter was done monthly for 6 months

- A single reference neurologist (CB) examined all patients at baseline and at 1 (median 30 days) after giving paclitaxel

- Two patients had paired exams conducted by a single neurologist at an outside institution

- Previous neurological assessments were not blinded to the examiner

- A detailed neurologic history was obtained, including possible risk factors for the development of peripheral neuropathy (diabetes, alcohol abuse, or prior history of neurotoxic chemotherapy or neuropathy)

- A peripheral neuropathy assessment instrument was used to facilitate and standardize data collection.

\section{Grading of neuropathy}

- Questions assessing the symptom of myalgia, arthralgia, numbness of toes and fingers, paresthesia, dysesthesia, was queried separately for fingers and toes and was graded according to NCI-CTC revised version 1999 into five grades (Grade 0 to Grade 5). The highest grade reported at anytime during 6 months by the patient was taken for analysis and comparison

- Grading of neuropathy was done according to NCI-CTC revised version 1999 into 4 grades (Grade 0-4) monthly for 6 months and examination done at 3 and 6 months was taken for analysis and comparison.

\section{Clinical examination of neuropathy}

- Clinical examination including vibration, grading of power in muscles, reflexes, cranial nerve examination, postural drop was done at baseline and monthly for 6 months. Examination findings at baseline, 3 and 6 months were taken for assessment and comparison

- Following clinical examination variables were taken for assessment:

1. Vibration at toes at baseline, 3 and 6 months

2. Vibration at ankles at baseline, 3 and 6 months

3. Knee reflexes at baseline, 3 and 6 months

4. Power in left and right dorsiflexors at baseline, 3 and 6 months

5. Cranial nerve weakness at baseline, 3 and 6 months

6. Postural drop baseline, 3 and 6 months.

\section{Electrophysiological studies}

All patients were evaluated by nerve-conduction studies at baseline, 3 and 6 months.

Motor and sensory responses were recorded using standardized equipment and techniques.

Previous electrophysiological assessments were not blinded.

Variables taken for assessment were:

1. Sensory nerve action potential (SNAP) of left and right sural nerve at baseline, 3 and 6 months

2. The distal latency of right and left sural nerve at baseline, 3 and 6 months

3. SNAP of right and left superior peroneal nerve at baseline, 3 and 6 months

4. Compound motor action potential (CMAP) of right and left common peroneal nerve at baseline, 3 and 6 months

5. CMAP of right and left posterior tibial nerve at baseline, 3 and 6 months.

\section{Statistical analysis}

Data were analyzed by using MS Excel and SPSS Statistics for Windows, version 15 (SPSS Inc., Chicago, Ill., USA). Quantitative data (age, cycles received, \% change in the mean of CMAP, SNAP and distal latencies) was expressed in terms of mean \pm standard deviation. Qualitative data (Neuropathy Grading, Symptom Grading-Myalgia, Arthralgia, Paresthesia, Dysesthesia, Numbness of toes and Fingers and Vibration sensation) was expressed as frequency and percentage.

Statistical significant change in quantitative variable was estimated by paired $t$-test. The proportion of qualitative variable in control and case was tested by Chi-square test. All the statistical tests performed at 5\% level of significance.

A value of $P<0.05$ considered as statistically significant.

\section{Results}

A total of 50 patients, aged 30 or more with diagnosis of 


\begin{tabular}{|c|c|c|c|c|c|c|}
\hline \multicolumn{5}{|c|}{ NCIC-CTC at 3 months after starting paclitaxel } & \multirow[t]{2}{*}{ Total } & \multirow[t]{2}{*}{$\boldsymbol{P}$} \\
\hline & Grade 0 & Grade 1 & Grade 2 & Grade 3 & & \\
\hline Glutamine, $n(\%)$ & $5(20.00)$ & $14(56.00)$ & $4(16.00)$ & $2(8.00)$ & $25(100.00)$ & $\chi^{2}=0.356$ \\
\hline Control, $n(\%)$ & $6(24.00)$ & $12(48.00)$ & $5(20.00)$ & $2(8.00)$ & $25(100.00)$ & $P=0.949$ \\
\hline Total, $n(\%)$ & $11(22.00)$ & $26(52.00)$ & $9(18.00)$ & $4(8.00)$ & $50(100.00)$ & \\
\hline
\end{tabular}

NCIC-CTC: National Cancer Institute-Common Toxicity scale

Table 2: Grade of neuropathy at 6 months with paclitaxel treatment

\begin{tabular}{lcccccc}
\hline & \multicolumn{1}{c}{ NCIC-CTC at 6 months after starting paclitaxel } & & Total & \\
\cline { 1 - 4 } Grade & $\mathbf{0}$ & $\mathbf{1}$ & $\mathbf{2}$ & $\mathbf{3}$ & & \\
\hline Glutamine & $5(20.00)$ & $13(52.00)$ & $5(20.00)$ & $2(8.00)$ & $25(100.00)$ & $\chi^{2}=0.458$ \\
Control & $5(20.00)$ & $11(44.00)$ & $6(24.00)$ & $3(12.00)$ & $25(100.00)$ & $P=0.938$ \\
Total, $n(\%)$ & $10(20.00)$ & $24(48.00)$ & $11(22.00)$ & $5(10.00)$ & $50(100.00)$ & \\
\hline
\end{tabular}

NCIC-CTC: National Cancer Institute-Common Toxicity scale

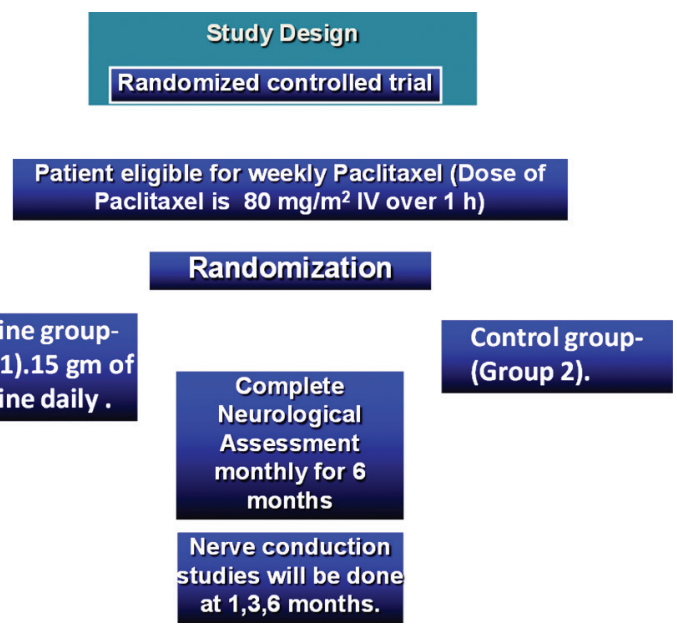

Figure 1: Study design

cancer and fulfilling the inclusion and exclusion criteria, formed the study population. Mean age was comparable between the both groups. The age distribution of the patients was included in this study. Most of the patients in this study were elderly aged $\geq 50$ years of age. Among them, 37 were female and 13 were male and were equally distributed between the groups.

Mean of number of chemotherapy cycles in both the groups was same. In glutamine group, chemotherapy was stopped in one patient and in control group chemotherapy was stopped in two patients due to neurotoxity. In glutamine group, Out of 50 patients, three patients had diabetes mellitus (DM) in each group and total patients with comorbidities were 8 out of 50 patients.

Neuropathy was graded as per NCI-CTC Scale and it was observed that $52 \%$ of patients developed Grade 1 neuropathy, $18 \%$ developed Grade 2 months and $8 \%$ developed Grade 3 neuropathy at 3 months. At 6 months, there was an increase in Grade 2 neuropathy by $4 \%$ and grade by 3 by $2 \%$. Results were comparable between the

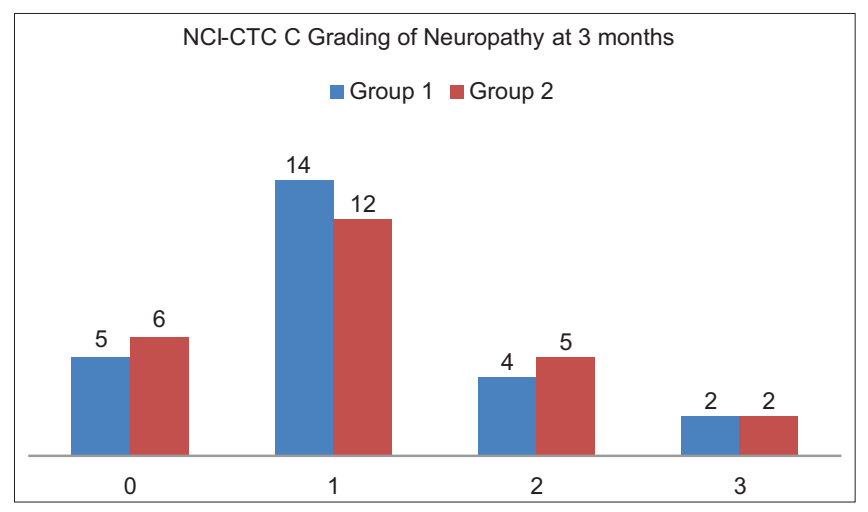

Figure 2: Paclitaxel-induced neuropathy as per $\mathrm{NCl}-\mathrm{CTC}$

groups at 3 months $(P=0.949)$ and 6 months $(P=0.938)$. There was no Grade 4 neuropathy in either of the groups [Tables 1, 2 and Figures 2, 3].

The incidence of neuropathy of all grades of neuropathy at 3 months was $78 \%$ and at 6 months was $80 \%$.

We graded symptoms of paclitaxel-induced neuropathy (Myalgias, Arthralgias, Paresthesias, Dysesthesias, Numbness of fingers, and Numbness of toes) with NCI-CTC Scale and assessment was done for the highest grade recorded by the patient during treatment for 6 months.

In this study, Grade 1 was the most common grade of symptom reported by the patient $(40 \%-50 \%) .2^{\text {nd }}, 3^{\text {rd }}$, and $4^{\text {th }}$ most common grade of symptom reported by the patient was Grade 0, Grade 2, and Grade 3, respectively. There was no Grade 4 symptom reported by the patient.

In this study, most common symptom reported was numbness in toes $(74 \%)$.

All the symptoms were statistically comparable in both groups (Myalgias: $P=0.066$, Arthralgia: $P=0.93$, Dysesthesia: $P=0.82$, Paresthesia: $P=0.92$, Numbness fingers: $P=0.97$, Numbness toes: $P=0.60)$. 
Vibration sense in Ankles was absent in 13 patients (26\%) at 6 months and vibration sensation in ankles was absent in $28(56 \%)$.

There was a trend toward less vibration loss in glutamine group, but at follow-up of 6 months, this advantage was lost. Results at 3 and 6 months were statistically comparable in toes as well as in ankles.

Only 1 patient had Grade 4 weakness in dorsiflexors at 3 months in glutamine group that persisted until 6 months. Results were statistically comparable at 3 months $(P=0.31)$ and 6 months $(P=1)$.

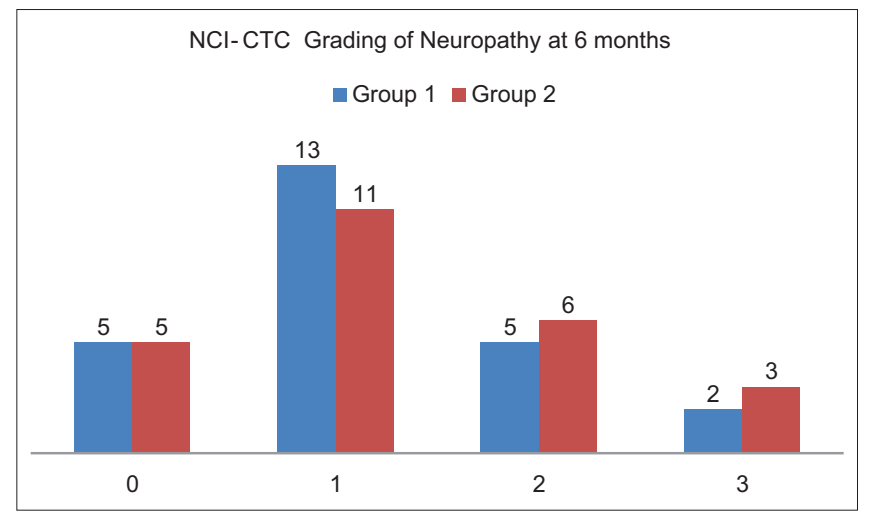

Figure 3: Grade of neuropathy at 6 months after paclitaxel treatment
Lower extremity reflexes were more likely to be preserved in both the groups. Only 5 patients had hyporeflexia at the end of 3 months in both groups which persisted until 6 months. Results were statistically comparable (At 3 months $P=0.69$ and 6 months $P=0.69)$.

In our study, there was no incidence of cranial nerve weakness or any incidence of the postural drop. One patient had a postural drop at the start of chemotherapy which persisted throughout the treatment and did not worsen during the treatment [Table 3 and Figure 4].

In our study, the percent changes of the mean SNAP amplitudes of right sural and left sural nerve before and after weekly paclitaxel treatment for both the control group and the glutamine group. The percent changes of the mean distal latency of left sural and right sural nerve before and after weekly paclitaxel treatment for both the control group and the glutamine group.

Percent change of the mean SNAP amplitude was more pronounced in the control group than the glutamine group for both right and left sural nerve at 3 months as well as 6 months but did not reach statistical significance.

Percentage change of the mean distal latency of left sural nerve at 3 months was more pronounced in control group,

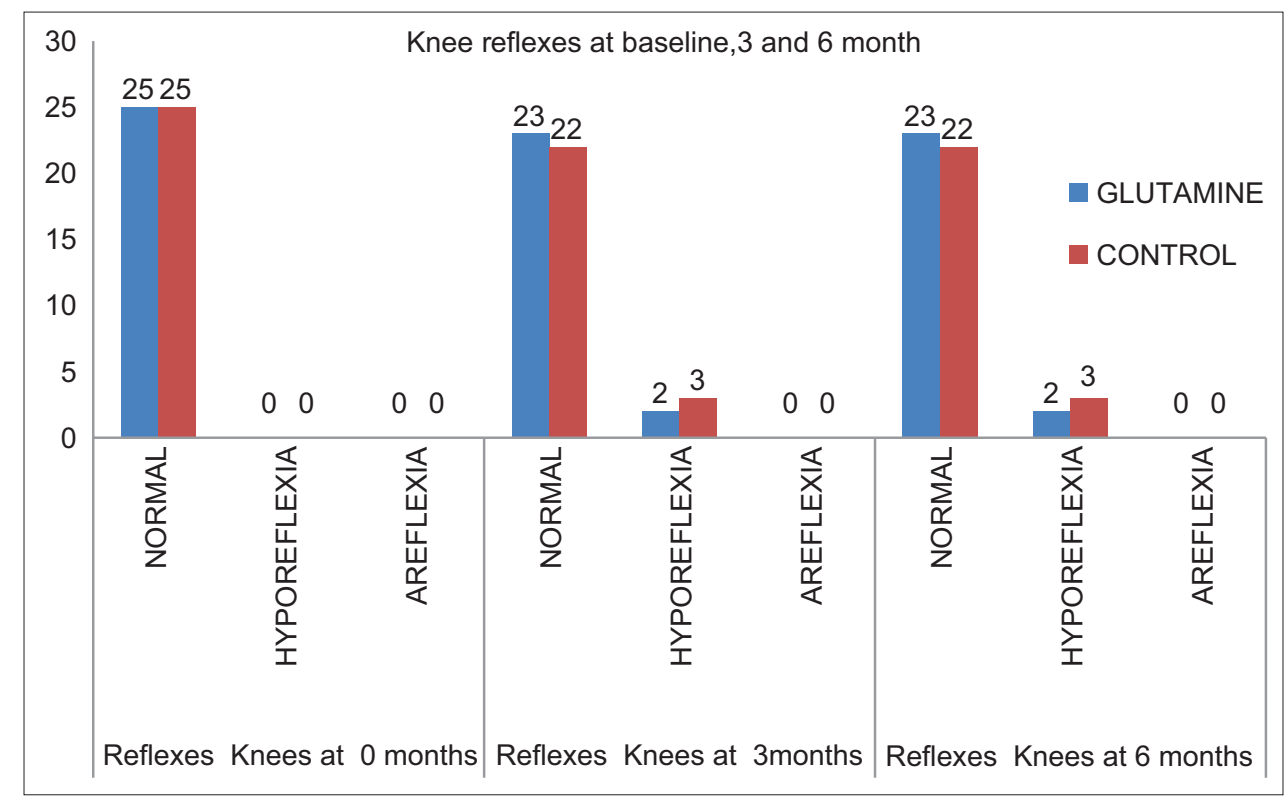

Figure 4: Knee reflexes at baseline, 3 and 6 months

Table 3: Knee reflexes at baseline, 3 and 6 months

\begin{tabular}{|c|c|c|c|c|c|c|c|c|c|c|c|}
\hline & \multicolumn{3}{|c|}{ Knee reflexes baseline } & \multicolumn{4}{|c|}{ Knee reflexes 3 months } & \multicolumn{4}{|c|}{ Knee reflexes 6 months } \\
\hline & Normal & Hyporeflexia & Areflexia & Normal & Hyporeflexia & Areflexia & $P$ & Normal & Hyporeflexia & Areflexia & $P$ \\
\hline Glutamine & 25 & 0 & 0 & 23 & 2 & 0 & $\chi^{2}=0.22$ & 23 & 2 & 0 & $\chi^{2}=0.22$ \\
\hline Control & 25 & 0 & 0 & 22 & 3 & 0 & $P=0.69$ & 22 & 3 & 0 & $P=0.69$ \\
\hline Total & 50 & 0 & 0 & 45 & 5 & 0 & & 45 & 5 & 0 & \\
\hline
\end{tabular}


Table 4: Percentage of mean SNAP between the two arms after paclitaxel

\begin{tabular}{|c|c|c|c|c|}
\hline & Glutamine & Control & Percentage difference & $P$ \\
\hline Left sural nerve SNAP 3 months & 20.66 & 23.82 & 3.16 & 0.51 \\
\hline Right superior peroneal Nerve 3 months & 10.83 & 28.83 & 5.46 & 0.1 \\
\hline Right superior peroneal Nerve 6 months & 24.53 & 29.44 & 4.9 & 0.1 \\
\hline Left superior peroneal nerve 6 months & 19.24 & 16.80 & 2.44 & 0.49 \\
\hline Left superior peroneal nerve 6 months & 24.91 & 22.67 & 2.24 & 0.54 \\
\hline Left sural nerve distal latency 3 months & 16.52 & 20.63 & 4.11 & 0.45 \\
\hline Left sural nerve distal latency 3 months & 26.54 & 22.66 & 3.88 & 0.35 \\
\hline Right sural nerve distal latency 3 months & 19.64 & 16.49 & 3.15 & 0.37 \\
\hline Right sural nerve distal latency 3 months & 27.13 & 24.82 & 2.31 & 0.52 \\
\hline
\end{tabular}

SNAP - Sensory nerve action potential

Table 5: Percentage of mean CMAP between the two arms after paclitaxel

\begin{tabular}{|c|c|c|c|c|}
\hline & Glutamine & Control & Percentage difference & $P$ \\
\hline \multicolumn{5}{|l|}{ CMAP } \\
\hline Left common peroneal nerve 3 months & 4.46 & 4.38 & 0.14 & 0.98 \\
\hline Left common peroneal nerve 6 months & 5.52. & 4.95 & 0.57 & 0.81 \\
\hline Right common peroneal nerve 3 months & 7.9 & 7.11 & 0.87 & 0.78 \\
\hline Right common peroneal nerve 6 months & 10.43 & 9.83 & 0.6 & 0.88 \\
\hline Left posterior tibial nerve 3 months & 6.01 & 5.80 & 0.21 & 0.95 \\
\hline Left posterior tibial nerve 6 months & 7.5 & 6.9 & 0.64 & 0.86 \\
\hline Right posterior tibial nerve 3 months & 3.34 & 3.13 & 0.23 & 0.83 \\
\hline Right posterior tibial nerve 6 months & 4.05 & 4.29 & 0.24 & 0.34 \\
\hline
\end{tabular}

CMAP - Compound muscle action potential

but at 6 months, it was more pronounced in glutamine group. Percentage change of mean distal latency of right sural nerve at 3 and 6 months was more pronounced in glutamine group at 3 and 6 months as well [Table 4].

In our study, percent change of the mean CMAP amplitude is more pronounced in glutamine group in left and right common peroneal nerve at 3 months (\% difference of 0.14 and 0.87 respectively), and at 6 months (\% difference 0.81 and 0.88 respectively).

Percentage change of the mean CMAP amplitude is also more pronounced in glutamine group in left posterior tibial nerve at 3 months (\% difference 0.95$), 6$ months $(0.86)$, and in right posterior tibial nerve ( $\%$ change 0.23 ). The percentage change of the mean CMAP amplitude is more pronounced in control group in right posterior tibial nerve at 6 months (\% difference 0.24$)$.

The percent changes in the average CMAP amplitudes between the control and glutamine groups did not reach statistical significance for any of the nerves tested [Table 5].

\section{Discussion}

The dose-limiting toxicity of many cancer chemotherapeutic agents is peripheral neuropathy. It is frequently a major detriment to quality of life in patients who have received and who are actively receiving neurotoxic chemotherapy. Few interventions can improve the symptoms and none can prevent it. Paclitaxel is the most common cause of neurotoxicity which is predominantly sensory. ${ }^{[2,3,15]}$ Weekly administration of paclitaxel induces a clear increase in dose-intensity, ${ }^{[16]}$ without significant enhancement of toxicities apart from neurotoxicity. Weekly paclitaxel causes more neurotoxicity than hematological and other non-hematological toxicities. There is more incidence of sensory neuropathy than motor neuropathy with weekly paclitaxel. Weekly paclitaxel infusions also result in more sensory neuropathy than 3 weekly infusions..$^{[17-20]}$

CIPN is most commonly assessed by grading scales developed by clinicians, including the National Cancer Institute Common Terminology Criteria for Adverse Events (NCI-CTCAE). ${ }^{[2]}$ NCS techniques remain the gold standard approach to assess clinical neuropathy and should be used in clinical trials of neuroprotective agents to provide objective evidence of nerve damage or neuroprotection. The drawback of NCS is that parameters may remain unchanged even until the end of chemotherapy. ${ }^{[21]}$ This proves the importance of neurological assessment by electrophysiological studies and clinically in clinical trials.

In the Indian context, no studies appear to have been conducted on detailed clinical and electrophysiological studies for the assessing the incidence of neuropathy with weekly paclitaxel.

With this background, we felt that our study could find answers to some important and little addressed questions like:

- The incidence of neuropathy with weekly paclitaxel in Indian context? 
- The pattern of neuropathy with weekly paclitaxel (sensory vs. motor) in Indian context?

- Clinical features of weekly paclitaxel-induced neuropathy?

- What are the important risk factors behind this?

- Can weekly paclitaxel-induced neuropathy be prevented with oral glutamine?

\section{Study population}

A total of fifty patients with a diagnosis of malignancy were included in the study. They were randomly assigned to one of the treatment arms. Patients assigned to glutamine group received daily glutamine ( $15 \mathrm{~g}$ /day) from the day of the start of paclitaxel for 6 months, irrespective of whether the patient has finished treatment protocol or not. Patients in the control group did not receive glutamine or any other supplementation apart from routine medication.

\section{Patient profile}

The age of the patients included in the study varied between 30-82 years. Most of the patients included in the study were elderly aged $\geq 50$ years of age ( 45 patients out of 50 ). As weekly paclitaxel is most commonly used in breast cancer and ovarian cancer, out of 50 patients, 37 were female and 13 were male.

In glutamine group, mean of a total number of chemotherapy, cycles were 16.15, whereas mean of chemotherapy cycles in control group was 16.36 , which was statistically comparable $(P=0.82)$.

In this study, there were only 8 patients with comorbidities. Six patients had DM and three in each group. All patients with DM developed Grade 1 neuropathy, suggesting that weekly paclitaxel does not cause more severe neuropathy in patients with DM. However, all the patients with DM had normal electrophysiological studies at baseline, no patient had received any neurotoxic chemotherapy prior, and patients with any neuropathy were excluded from study, therefore, this study was limited to address our secondary objective "to determine the risk factors for weekly paclitaxel-induced neuropathy." This may suggest that patients with DM and patients with prior chemotherapies will not have exaggerated neurotoxicity with paclitaxel if their baseline electrodiagnostic studies are normal but that needs to be addressed in a larger study.

The incidence of neuropathy with weekly paclitaxel: in our study, at 6 months, $20 \%, 48 \%, 22 \%$, and $10 \%$ of patients

\begin{tabular}{|c|c|c|c|}
\hline \multicolumn{4}{|c|}{ Table 6: Incidence of neuropathy with weekly paclitaxel } \\
\hline \multicolumn{2}{|c|}{ At 3 months } & \multicolumn{2}{|c|}{ At 6 months } \\
\hline Grade \% & Total $(78 \%)$ & Grade \% & Total $(80 \%)$ \\
\hline Grade 0 & $22 \%$ & Grade 0 & $20 \%$ \\
\hline Grade 1 & $52 \%$ & Grade 1 & $48 \%$ \\
\hline Grade 2 & $18 \%$ & Grade 2 & $22 \%$ \\
\hline Grade 3 & $8 \%$ & Grade 3 & $10 \%$ \\
\hline Grade 4 & 0 & Grade 4 & 0 \\
\hline
\end{tabular}

had Grade 0, 1, 2, and 3 grade neuropathy respectively. Same results were seen in a study of weekly paclitaxel in breast cancer by Sparano et al. ${ }^{[19]}$ comparing paclitaxel with docetaxel. He reported the highest incidence of neuropathy with weekly paclitaxel, $6 \%$ of Grade 3, 4 neuropathy. The study also showed $8 \%$ of Grade 3 neuropathy at 3 months and $10 \%$ at 6 months. In the same study combined incidence of Grade 2, 3, and Grade 4 neuropathy was $27 \%$ and in our study incidence of Grade 2, 3 and Grade 4 neuropathy was $26 \%$ at 3 months and $32 \%$ at 6 months. There was no Grade 4 neuropathy in our study. This shows the incidence of neuropathy with weekly paclitaxel in the Indian context is comparable with others [Table 6].

In another study done by Seidman et al., ${ }^{[17]}$ incidence of neuropathy with weekly paclitaxel given in metastatic breast cancer was $24 \%$ of Grade 3 , and only 1 patient developed Grade 4 sensory peripheral neuropathy. In our study, no patient had Grade 4 neuropathy showing less incidence of Grade 4 neuropathy with weekly paclitaxel in our patients. But the incidence of Grade 3 neuropathy being more than our study ( $10 \%$ at 6 months) could be attributed to that every patient had received one chemotherapy regimen before paclitaxel.

The total incidence of neuropathy including all grades in our study was $78 \%$ at 3 months and $80 \%$ at 6 months.

Results in both groups were comparable at 3 and 6 months proving no role of glutamine in the prevention of CIPN.

\section{Symptoms of neuropathy}

Trials done in the past with Glutamine for prevention of Paclitaxel-induced have not graded peripheral neuropathy as per uniform criteria and NCIC-CTC grading scale ${ }^{[22-28]}$ except study done by Wang et al. ${ }^{[29]}$ used NCI-CTC Criteria, although in this study glutamine was used for prevention of oxaliplatin-induced neuropathy. Most commonly used scales for assessing neuropathy are NCI-CTC and WHO but still percentage interobserver agreement across all grades of severity ranged from 45 (NCI-CTC) to 83 (WHO). Reliability increases with training. In our study, we used NCI-CTC for grading neuropathy and its symptoms [Table 7].

The goal of giving patients glutamine was to decrease the myalgias, and that was observed in patients treated with high dose paclitaxel by Severase et al. ${ }^{[10]}$ Glutamine will decrease the neuropathy was not expected, and it was an unanticipated observation. In another study by Stubblefield et $a l .^{[13]}$ glutamine had great impact in reducing the signs of neuropathy like finger numbness $(P=0.06)$ and toe numbness $(P=0.04)$. Moderate to severe paresthesia were also observed less frequently in the glutamine group although value was not statistically significant. Limitations of these studies were they were not randomized trials, patients were unevenly distributed in study groups. Moreover, these studies were done with very high dose 
Table 7: Grading and comparison of symptoms National Cancer Institute Common Toxicity scale grading and comparison of symptoms induced by paclitaxel (highest grade recorded during study)

\begin{tabular}{|c|c|c|c|c|c|c|}
\hline & Grade 0 & Grade 1 & Grade 2 & Grade 3 & Grade 4 & $P$ \\
\hline \multicolumn{7}{|l|}{ Myalgia } \\
\hline Glutamine & 10 & 10 & 4 & 1 & 0 & \multirow[t]{2}{*}{0.066} \\
\hline Control & 4 & 13 & 5 & 3 & 0 & \\
\hline \multicolumn{7}{|l|}{ Arthralgia } \\
\hline Glutamine & 3 & 17 & 3 & 2 & 0 & \multirow[t]{2}{*}{0.93} \\
\hline Control & 11 & 8 & 3 & 3 & 0 & \\
\hline \multicolumn{7}{|l|}{ Dysesthesia } \\
\hline Glutamine & 6 & 12 & 5 & 2 & 0 & \multirow[t]{2}{*}{0.822} \\
\hline Control & 8 & 9 & 5 & 3 & 0 & \\
\hline \multicolumn{7}{|l|}{ Paresthesia } \\
\hline Glutamine & 7 & 12 & 4 & 2 & 0 & \multirow[t]{2}{*}{0.920} \\
\hline Control & 7 & 10 & 5 & 3 & 0 & \\
\hline \multicolumn{7}{|c|}{ Numbness fingers } \\
\hline Glutamine & 7 & 11 & 5 & 2 & 0 & \multirow[t]{2}{*}{0.970} \\
\hline Control & 7 & 10 & 5 & 3 & 0 & \\
\hline \multicolumn{7}{|c|}{ Numbness toes } \\
\hline Glutamine & 7 & 12 & 5 & 1 & 0 & \multirow[t]{2}{*}{0.607} \\
\hline Control & 6 & 9 & 7 & 3 & 0 & \\
\hline
\end{tabular}

paclitaxel. The study by Loven et al..$^{[28]}$ showed glutamine failed to prevent neuropathy of weekly paclitaxel.

In our study, highest grade of symptoms of paclitaxel-induced neuropathy during 6 months was taken for assessment and comparison. The incidence of Grade 1 was highest $(42 \%-50 \%) .2^{\text {nd }}$ and $3^{\text {rd }}$ most common grade across all symptoms was Grade $2(12 \%-24 \%)$ and Grade 3 $(8 \%-10 \%)$. The total incidence of all grades across all symptoms was $72 \%-74 \%$. The incidence of Grade 2 and Grade 3 symptoms of numbness of toes and fingers in glutamine was less but statistically comparable. There were no Grade 4 symptoms reported by any patient.

Our results differ with the studies mentioned earlier, ${ }^{[10,13]}$ which showed the protective role of glutamine for prevention of CIPN where a higher dose of paclitaxel was used in transplant settings and neurotoxicity profile does differ between the high dose and weekly paclitaxel.

A pilot study done by Loven et al..$^{[5,28]}$ where dose weekly paclitaxel was also used as was in our study, did not show any protective role glutamine in preventing CIPN as did our study. Results showed glutamine has no role in the prevention of Paclitaxel-induced symptoms of neuropathy.

\section{Signs of neuropathy (neurological examination)}

In a study by Stubblefield et al. ${ }^{[13]}$ vibratory sensation was much worse in $42 \%$ in control group and $24 \%$ in glutamine group. Vibration sense was worse in $54 \%$ patients in control group and $45 \%$ in glutamine group making it statistically significant (0.04). A study done by Loven. et al. ${ }^{[28]}$ with paclitaxel showed there was less vibration sensation loss in glutamine group but not statistically significant, proving glutamine could not prevent paclitaxel-induced neuropathy. In our study, at 3 months vibration sense in toes was absent in $20 \%$ of patients in glutamine group and $24 \%$ of patients in control group, and at 6 months, vibration sense was absent in $28 \%$ of patients in glutamine group and $24 \%$ of patients in the control group. Again showing a different neurotoxicity profile with weekly paclitaxel with less number of patients developing loss of vibration sensation. Loss of vibration sensation in toes and ankles at 3 and 6 months was statistically comparable. This showed that glutamine could not prevent loss of vibration sense in patients receiving weekly paclitaxel.

In a study by Vahdat et al. ${ }^{[27]}$ less motor weakness was also observed in patients who received glutamine as compared with patients in the control group $(P=0.04)$. However, the study done by Loven et al. ${ }^{[28]}$ with weekly paclitaxel could not show a significant difference in the decreased frequency of motor weakness. In our trial, we also could not find any statistically significant difference between the power in Ankle Dorsiflexors between both groups when assessed at 3 and 6 months.

There was only one patient with Grade 4 power in the right and left dorsiflexors at 3 and 6 months and one patient with a decrease in power in right quadriceps at 3 and 6 months making interpretation and comparison difficult. Rest of patients had normal power during assessment for 6 months. In a study by Vahdat et al. ${ }^{[27]} 22$ out of 45 patients had some grade of motor weakness because patients received a very high dose of paclitaxel. This concludes weekly paclitaxel is not a frequent cause of motor neuropathy, showing different neurotoxicity profile between the high and dose-dense paclitaxel.

We also assessed and compared other signs of neuropathy in our patients including reflexes, postural drop, and cranial 
nerve weakness. Only 2 patients in glutamine group and 3 patients in control group had hyporeflexia, whereas in a study done by Vahdat et al. ${ }^{[27]} 71 \%$ patients in the control group and $50 \%$ in glutamine group had hyporeflexia, proving weekly has different neurotoxicity profile than high dose paclitaxel. During treatment course, no patient had cranial nerve palsy of any kind and only one patient had a postural drop at the beginning of the treatment in both groups, and there was no increase in his postural drop of blood pressure during the course the treatment.

\section{Electrophysiological studies}

The percentage change in the mean SNAP, CMAP amplitudes and velocities of sensory (Sural and superior peroneal nerve), motor (peroneal, tibial), and distal latencies (sural nerve) after weekly paclitaxel treatment for both the control group and the glutamine group.

In a study done by Loven $\mathrm{D}$ et al. ${ }^{[28]}$ percentage change of mean SNAP was variable across the nerves, and percentage change of mean amplitudes between the control and glutamine group did not reach statistical significance for any of the nerves tested. In our study, less decrement in CMAP was seen in both the groups as compared to SNAP and distal latency. Percentage change in CMAP was also less across all nerves proving weekly paclitaxel causes predominant sensory neuropathy. The percentage change mean SNAP, CMAP, distal latency at 3 months and 6 months between the groups did vary whether sensory or motor but no result reached statistical significance, showing glutamine does not confer the protective effect for prevention of neurotoxicity induced by weekly paclitaxel [Tables 4 and 5].

There were significant decrements in the amplitude and conduction velocity of the sensory (sural and superior peroneal nerve), motor (peroneal, tibial,) and) and distal latencies (Sural nerve) from the baseline in all patients despite the fact that whether patient has symptoms of neuropathy or not, showing that all patients develop sub-clinical neuropathy that will be detected by electrophysiological studies. However, unfortunately, no grading system for electrodiagnostic studies is available for CIPN, it becomes difficult to interpret the results and severity as per electrodiagnostic studies.

\section{Conclusion}

1. Totally 50 patients were enrolled in this study. Patients were randomized into two groups by simple randomization, 25 patients in each group. Patients in the glutamine group received oral glutamine of $15 \mathrm{~g} /$ day from the start of treatment for 6 months. Control group did not receive any treatment apart from routine medications

2. In our study, we graded neuropathy and symptoms as per national cancer institute common toxicity scale most commonly used toxicity scale to assess CIPN, which has least interobserver variability

3. Subclinical peripheral neuropathy due to chemotherapy can be evaluated with the use of quantitative assessments like nerve conduction velocity

4. The electrophysiological study is the best tool available to diagnose neuropathy and can detect neuropathy at the very earlier stage even when the clinical exam is negative. Apart from that nature of neuropathy can be determined but grading is not possible which makes very difficult to decide on follow-up examinations when the physician should intervene. Moreover, there are fluctuations in SNAP and CMAP, and these fluctuations are most probably related to the innate variability of serial nerve conduction study parameters, particularly motor and sensory amplitude

5. There were significant decrements in amplitude, velocity, and distal latencies recorded by electrophysiological studies. More decrements were seen in the sensory nerves. But glutamine did not appear to exert a protective effect

6. Electrophysiological studies showed decrements in amplitudes and distal latencies in all patients, but $20 \%$ of patients did not develop clinical neuropathy at the end of 6 months suggesting clinical findings and electrophysiological findings may not correlate

7. The total incidence of neuropathy including all grades in our study was $78 \%$ at 3 months and $80 \%$ at 6 months

8. The incidence of Grade 1 neuropathy was $48 \%$, Grade 2 was $22 \%$, and Grade 3 was $10 \%$ at 6 months. About 20\% did not develop any neuropathy. Symptoms of weekly paclitaxel were highest of Grade 1 and 2, and there were no Grade 4 symptoms, and all symptoms were statistically comparable between the treatment arms

9. The incidence of motor neuropathy was very less with only two patients developing Grade 4 weaknesses, one in dorsiflexors and another one in quadriceps. Results were comparable in both the groups. This suggests neuropathy with weekly paclitaxel is predominantly sensory and there is less incidence of motor neuropathy as compared to high dose paclitaxel

10. In studies of high dose paclitaxel, there are higher incidences of motor neuropathy, but our study showed same is not true with weekly paclitaxel which causes significant sensory neuropathy and has a very little impact on motor neuropathy

11. At last, glutamine did not prevent neurotoxicity induced by weekly paclitaxel.

Financial support and sponsorship

Nil.

\section{Conflicts of interest}

There are no conflicts of interest. 


\section{References}

1. Hausheer FH, Schilsky RL, Bain S, Berghorn EJ, Lieberman F. Diagnosis, management, and evaluation of chemotherapy-induced peripheral neuropathy. Semin Oncol 2006;33:15-49.

2. Lipton RB, Apfel SC, Dutcher JP, Rosenberg R, Kaplan J, Berger A, et al. Taxol produces a predominantly sensory neuropathy. Neurology 1989;39:368-73.

3. Rowinsky EK, Chaudhry V, Cornblath DR, Donehower RC. Neurotoxicity of Taxol. J Natl Cancer Inst Monogr 1993;15:107-15.

4. Bitton RJ, Figg WD, Reed E. A preliminary risk-benefit assessment of paclitaxel. Drug Saf 1995;12:196-208.

5. Freilich RJ, Balmaceda $C$, Seidman AD, Rubin $M$, DeAngelis LM. Motor neuropathy due to docetaxel and paclitaxel. Neurology 1996;47:115-8.

6. Eisenhauer EA, ten Bokkel Huinink WW, Swenerton KD, Gianni L, Myles J, van der Burg ME, et al. European-Canadian randomized trial of paclitaxel in relapsed ovarian cancer: High-dose versus low-dose and long versus short infusion. J Clin Oncol 1994;12:2654-66.

7. Smith RE, Brown AM, Mamounas EP, Anderson SJ, Lembersky BC, Atkins JH, et al. Randomized trial of 3-hour versus 24-hour infusion of high-dose paclitaxel in patients with metastatic or locally advanced breast cancer: National surgical adjuvant breast and bowel project protocol B-26. J Clin Oncol 1999;17:3403-11.

8. Mielke S, Mross K, Gerds TA, Schmidt A, Wäsch R, Berger DP, et al. Comparative neurotoxicity of weekly non-break paclitaxel infusions over 1 versus 3 h. Anticancer Drugs 2003;14:785-92.

9. Kottschade LA, Sloan JA, Mazurczak MA, Johnson DB, Murphy BP, Rowland KM, et al. The use of Vitamin E for the prevention of chemotherapy-induced peripheral neuropathy: Results of a randomized phase III clinical trial. Support Care Cancer 2011;19:1769-77.

10. Savarese D, Boucher J, Corey B. Glutamine treatment of paclitaxel-induced myalgias and arthralgias. $J$ Clin Oncol 1998;16:3918-9.

11. Kautio AL, Haanpää M, Leminen A, Kalso E, Kautiainen H, Saarto $\mathrm{T}$, et al. Amitriptyline in the prevention of chemotherapy-induced neuropathic symptoms. Anticancer Res 2009;29:2601-6.

12. Rao RD, Michalak JC, Sloan JA, Loprinzi CL, Soori GS, Nikcevich DA, et al. Efficacy of gabapentin in the management of chemotherapy-induced peripheral neuropathy: A phase 3 randomized, double-blind, placebo-controlled, crossover trial (N00C3). Cancer 2007;110:2110-8.

13. Stubblefield MD, Vahdat LT, Balmaceda CM, Troxel AB, Hesdorffer CS, Gooch CL, et al. Glutamine as a neuroprotective agent in high-dose paclitaxel-induced peripheral neuropathy: A clinical and electrophysiologic study. Clin Oncol (R Coll Radiol) 2005;17:271-6.

14. Postma TJ, Vermorken JB, Liefting AJ, Pinedo HM, Heimans JJ. Paclitaxel-induced neuropathy. Ann Oncol 1995;6:489-94.

15. Pace A, Bove L, Aloe A, Nardi M, Pietrangeli A. Paclitaxel neurotoxicity: Clinical and neur18, Princeton NJ: Bristol-Myers Squibb. Taxol Prescribing Information. pathophysiologic study of
23 patients. Ital J Neurol Sci 1997;18:73-9.

16. Taxol Prescribing Information. Princeton NJ: Bristol-Myers Squibb; 2011.

17. Seidman AD, Berry D, Cirrincione C, Harris L, Muss H, Marcom PK, et al. Randomized phase III trial of weekly compared with every-3-weeks paclitaxel for metastatic breast cancer, with trastuzumab for all HER-2 overexpressors and random assignment to trastuzumab or not in HER-2 nonoverexpressors: Final results of Cancer and Leukemia Group B protocol 9840. J Clin Oncol 2008;26:1642-9.

18. Katsumata N, Yasuda M, Takahashi F, Isonishi S, Jobo T, Aoki D, et al. Dose-dense paclitaxel once a week in combination with carboplatin every 3 weeks for advanced ovarian cancer: A phase 3, open-label, randomised controlled trial. Lancet 2009;374:1331-8.

19. Sparano JA, Wang M, Martino S, Jones V, Perez EA, Saphner T, et al. Weekly paclitaxel in the adjuvant treatment of breast cancer. N Engl J Med 2008;358:1663-71.

20. Pace A, Nisticò C, Cuppone F, Bria E, Galiè E, Graziano G, et al. Peripheral neurotoxicity of weekly paclitaxel chemotherapy: A schedule or a dose issue? Clin Breast Cancer 2007;7:550-4.

21. Kiernan MC, Burke D, Andersen KV, Bostock H. Multiple measures of axonal excitability: A new approach in clinical testing. Muscle Nerve 2000;23:399-409.

22. Grothey A, Nikcevich DA, Sloan JA, Kugler JW, Silberstein PT, Dentchev $\mathrm{T}$, et al. Intravenous calcium and magnesium for oxaliplatin-induced sensory neurotoxicity in adjuvant colon cancer: NCCTG N04C7. J Clin Oncol 2011;29:421-7.

23. Wen F, Zhou Y, Wang W, Hu QC, Liu YT, Zhang PF, et al. $\mathrm{Ca} / \mathrm{Mg}$ infusions for the prevention of oxaliplatin-related neurotoxicity in patients with colorectal cancer: A meta-analysis. Ann Oncol 2013;24:171-8.

24. Loprinzi CL, Qin R, Dakhil SR, Fehrenbacher L, Flynn KA, Atherton $\mathrm{P}$, et al. Phase III randomized, placebo-controlled, double-blind study of intravenous calcium and magnesium to prevent oxaliplatin-induced sensory neurotoxicity (N08CB/Alliance). J Clin Oncol 2014;32:997-1005.

25. Argyriou AA, Chroni E, Polychronopoulos P, Iconomou G, Koutras A, Makatsoris T, et al. Efficacy of oxcarbazepine for prophylaxis against cumulative oxaliplatin-induced neuropathy. Neurology 2006;67:2253-5.

26. Rao RD, Flynn PJ, Sloan JA, Wong GY, Novotny P, Johnson DB, et al. Efficacy of lamotrigine in the management of chemotherapy-induced peripheral neuropathy: A phase 3 randomized, double-blind, placebo-controlled trial, N01C3. Cancer 2008;112:2802-8.

27. Vahdat L, Papadopoulos K, Lange D, Leuin S, Kaufman E, Donovan $\mathrm{D}$, et al. Reduction of paclitaxel-induced peripheral neuropathy with glutamine. Clin Cancer Res 2001;7:1192-7.

28. Loven D, Levavi H, Sabach G, Zart R, Andras M, Fishman A, et al. Long-term glutamate supplementation failed to protect against peripheral neurotoxicity of paclitaxel. Eur J Cancer Care (Engl) 2009;18:78-83.

29. Wang WS, Lin JK, Lin TC, Chen WS, Jiang JK, Wang HS, et al. Oral glutamine is effective for preventing oxaliplatin-induced neuropathy in colorectal cancer patients. Oncologist 2007;12:312-9. 